$>$ Nous poursuivons dans ce numéro la publication des Nouvelles rédigées par les étudiants du Master «Biologie Santé », dans le cadre du module «physiopathologie de la signalisation » de l'université Paris-Saclay. Ces textes s'inscrivent dans le développement d'un partenariat que médecine/ sciences propose aux Écoles doctorales (voir à ce propos l'Éditorial de Hervé Chneiweiss, $\mathrm{m} / \mathrm{s}$ $n^{\circ}$ 6-7, juin-juillet 2016, page 537). Après les quatre Nouvelles publiées dans le numéro de juinjuillet 2016, précédées d'une introduction des enseignants participants, et les cinq Nouvelles publiées dans le numéro d'août-septembre 2016, voici une troisième série de cinq Nouvelles. Toutes ces Nouvelles sont en accès libre sur le site de $\mathrm{m} / \mathrm{s}$ (www.medecine/ sciences.org). Bonne lecture! <
Partenariat médecine/sciences Écoles doctorales (3)

L'actualité scientifique vue par les étudiants du Master Biologie Santé, module physiopathologie de la signalisation, Université Paris-Saclay

\begin{tabular}{|c|c|c|}
\hline $\begin{array}{l}\text { UnIVersIte } \\
\text { PARIS-SACLAY }\end{array}$ & $\begin{array}{l}\text { SCHOOL } \\
\text { BIOLOGIE, MÉDECINE, } \\
\text { PHARMACIE }\end{array}$ & $\begin{array}{l}\text { MASTER } \\
\text { Biologie Santé }\end{array}$ \\
\hline
\end{tabular}

Karim Benihoud (professeur, université Paris-Sud) Sophie Dupré (maître de conférences, université Paris-Sud) Olivier Guittet (maître de conférences, université Paris-Sud) Boris Julien (maître de conférences, université Paris-Sud) Hervé Le Stunff (professeur, université Paris-Sud) Philippe Robin (maître de conférences, université Paris-Sud) karim.benihoud@u-psud.fr

Série coordonnée par Laure Coulombe

\title{
NOUVELLE
}

\section{La barrière de diffusion, une nouvelle fenêtre pour l'exclusion}

Coralie Alexandre $^{1}$, Stéphane Baillard ${ }^{1}$, Julien Tavet ${ }^{1}$, Oliver Nüsse ${ }^{2}$

\author{
${ }^{1}$ Ml Biologie Santé, Université Paris-Saclay, 91405 Orsay, \\ France ; \\ ${ }^{2}$ Laboratoire de chimie physique, UMR8000, \\ Université Paris-Sud, CNRS, Université Paris-Saclay, \\ 91405 Orsay, France. \\ coralie.alexandre@u-psud.fr \\ stephane.baillard@u-psud.fr \\ julien.tavet@u-psud.fr \\ oliver.nusse@u-psud.fr
}

> Nous vivons dans un monde dangereux où nous sommes en permanence agressés par des agents pathogènes (virus, bactéries, etc.), et c'est pour nous protéger que nous avons un système immunitaire, composé de l'immunité adaptative et de l'immunité innée. L'immunité innée n'est autre que la première ligne de défense, déjà en place avant la rencontre avec l'élément pathogène. Ses principaux

Cette Nouvelle fait partie d'une série de 15 Nouvelles rédigées par les étudiants du Master «Biologie Santé » de l'université Paris-Saclay qui paraîtront dans les numéros $6-7,8-9$ et 10 (2016) de médecine/sciences. acteurs sont les phagocytes - les macrophages et les polynucléaires neutrophiles -, qui, en ingérant divers agents pathogènes, protègent l'organisme.

\section{La synapse phagocytaire}

La phagocytose consiste à capturer et internaliser des débris cellulaires, voire des cellules entières (bactéries, champignons). Une fois les pathogènes dans la vésicule d'endocytose appelée phagosome, le milieu vésiculaire s'acidifie et le phagosome fusionne avec un lysosome pour former le phagolysosome, dont le rôle est de détruire l'élément pathogène. Le processus de phagocytose débute par un contact physique étroit entre la particule cible et le macrophage, appelé synapse phagocytaire par analogie avec les synapses neuronales et immunologiques. La formation de la synapse peut être déclenchée par les récepteurs Fc $\gamma R$, exprimés à la surface des macrophages [1] et qui reconnaissent la partie constante, $\mathrm{Fc}$, des immunoglobulines $\mathrm{G}$ ( $\operatorname{gg} \mathrm{G})$. Ces IgG vont recouvrir la bactérie pour qu'elle soit plus facilement reconnue par les macrophages, c'est le processus dit d'opsonisation. 


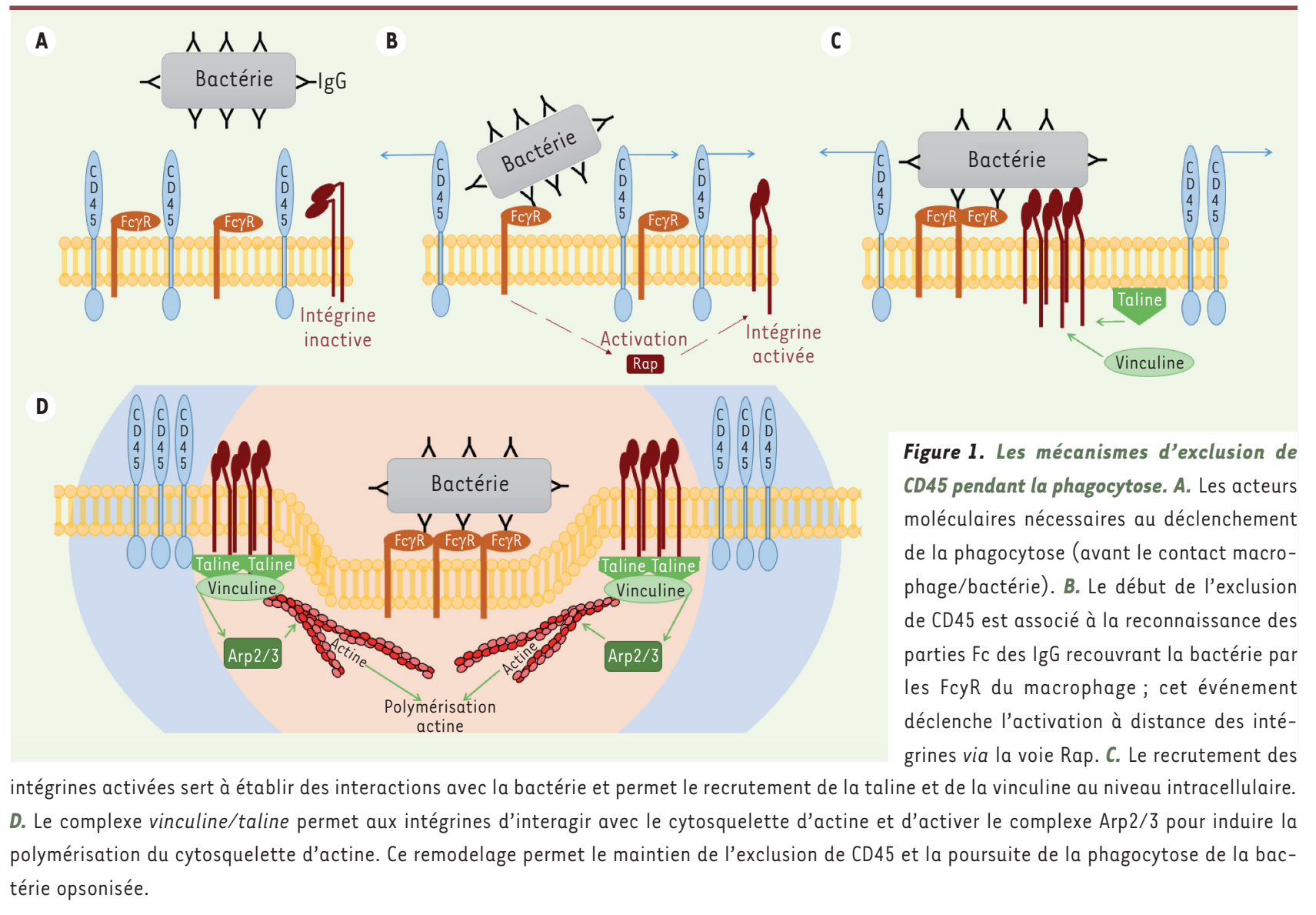

Parallèlement à ces interactions, plusieurs processus surviennent au niveau de la synapse phagocytaire: d'une part, une activation des tyrosine kinases de la famille des Src, et, d'autre part, une exclusion de la synapse phagocytaire des tyrosine phosphatases transmembranaires telles que les molécules CD45 (Figure 1). La phosphorylation des tyrosines étant indispensable au déclenchement de la phagocytose, l'exclusion de CD45 est un moyen de prolonger les signaux activateurs de la phagocytose $[2,3]$. Ces phosphatases sont aussi exclues des synapses immunologiques à cause de leur ectodomaine trop important pour la fente synaptique, où interagissent des récepteurs mobiles dans les membranes cellulaires. En revanche, durant la phagocytose, la mobilité des IgG recouvrant la bactérie n'est pas possible en raison de la rigidité de la paroi bactérienne. Par conséquent, l'exclusion des phosphatases CD45 par la taille de l'ectodomaine est-elle possible face à une proie rigide?

Afin d'élucider les mécanismes d'exclusion de CD45 de la synapse phagocytaire, Freeman et al. emploient une série de techniques sophistiquées de microscopie, de marquages moléculaires et de modèles cellulaires [2]. Ils réalisent des constructions (courte et longue) de glycopolymères synthétiques d' $\alpha$-N-acétylglycosamine (mimant le volume occupé par l'ectodomaine de CD45) marqué avec Alexa 488 (un fluorochrome). Ils réalisent également des constructions chimériques fusionnant les domaines transmembranaire et intracytoplasmique de CD45 avec le domaine extracellulaire soit de CD2 (de taille plus courte que celui de CD45) soit de CD43 (de taille équivalente à celui de CD45). Ils montrent ainsi que c'est la taille du domaine extracellulaire de CD45 qui est responsable de son exclusion de la synapse phagocytaire, et que seule la fusion CD45-CD43 permet la phagocytose.
Comment et pourquoi CD45 se retrouve hors-jeu?

Pour répondre à cette question, il est tout d'abord nécessaire de savoir comment CD45 se déplace au sein de la membrane plasmique d'un macrophage. Des molécules de CD45 exprimées à la membrane de ces macrophages ont été marquées individuellement par un anticorps (fragment Fab) anti-CD45 étiqueté avec un quantum $\operatorname{dot}^{1}$, et les cellules déposées sur des surfaces recouvertes d'IgG, qui, en se liant aux Fc $\gamma R$, déclenchent le processus. Les déplacements de chaque molécule CD45 ont été enregistrés (c'est la méthode dite du single molecule tracking). II en résulte l'identification de différentes trajectoires pour CD45 au sein de la membrane plasmique, isotropes ${ }^{2}$,

\footnotetext{
${ }^{1}$ Structure fluorescente nanométrique de semi-conducteur. ${ }^{2}$ Diffusion isotrope: mode de diffusion dans toutes les directions.
} 
par diffusion libre 3 ou confinée 4 , mais pas de trajectoire linéaire. La formation de la synapse augmente la proportion de déplacements par diffusion libre, qui atteint $70 \%$, indiquant une perte du confinement partiel du CD45, due à la rupture de l'organisation du cytosquelette d'actine. Ces CD45 se déplacent en périphérie de la synapse phagocytaire, créant une zone centrale dépourvue de CD45 au sein de cette synapse. Une fois en périphérie, les molécules CD 45 ne peuvent plus revenir au centre parce qu'une barrière de diffusion progressive a été mise en place autour de la synapse phagocytaire, à laquelle les molécules CD45 se heurtent et sur laquelle elles rebondissent. D'autres barrières de diffusion [4] existent dans de nombreuses cellules, comme dans les épines dendritiques, qui contrôlent la densité de récepteurs pour la transmission synaptique [5]. Toutefois, le fonctionnement moléculaire et la nature exacte de la barrière de diffusion dans la synapse phagocytaire ne sont pas bien compris. II a été suggéré par Freeman et al. que les intégrines pouvaient intervenir. En effet, les intégrines sont des récepteurs cellulaires d'adhésion transmembranaires, exprimés à la surface des macrophages (et de beaucoup d'autres cellules), et qui doivent être activés pour exercer leur fonction. Elles pourraient, avec leur partie extracellulaire, interagir directement avec le pathogène et de multiples autres ligands, et, via leur partie intracellulaire, avec le cytosquelette d'actine. La question qui se pose alors est: quelles sont les relations qui s'établissent entre les Fc $\gamma R$, les intégrines, le cytosquelette, et les tyrosine phosphatases CD45 au début de la phagocytose?

Mais qui sont les chefs d'orchestre du déclenchement de la phagocytose? Au sein de cette barrière, différentes molécules doivent intervenir et être

\footnotetext{
${ }^{3}$ Diffusion libre : mode de diffusion sans barrières.

${ }^{4}$ Diffusion confinée : les molécules diffusent dans une zone restreinte de l'espace membranaire.
}

exprimées à la surface du macrophage. Pour identifier chacune d'entre elles, il est nécessaire de les marquer via un anticorps spécifique, et d'utiliser des inhibiteurs qui leur sont propres. C'est ce qui a été exploité lors d'expériences de phagocytose «frustrée», qui permettent l'étude uniquement du déclenchement de la phagocytose et non du processus complet. En d'autres termes, l'élément à phagocyter n'est jamais totalement endocyté. Cette expérience est réalisée en déposant des spots d'IgG (micropatterning) sur une plaque de verre, distants de 2, 4, ou $6 \mu \mathrm{m}$, autour desquels est étalé ou non du PEG (polyéthylène glycol). Des macrophages sont ensuite déposés sur ces plaques et observés au microscope. Ces macrophages reconnaissent les spots d'IgG comme ils le feraient pour une bactérie couverte d'IgG (opsonisée) et forment une synapse phagocytaire.

Afin d'élucider les relations entre les $F c \gamma R$, les intégrines, les éléments du cytosquelette (réseau d'actine, taline, vinculine), ainsi qu'avec les CD45 au cours de ce processus, les auteurs [2] ont utilisé tout un arsenal de techniques physiques et microscopiques (MSS [moment scaling spectrum], pdot [single-qdot tracking], SIM [secondary ion mass spectrometry], TIRF [total internal reflection microscopy]).

La cinétique d'établissement des interactions entre $F c \gamma R$, intégrines, cytosquelette et CD45 a été définie: tout d'abord, les $F c \gamma R$ reconnaissent les parties Fc des IgG sur la plaque de verre et se regroupent uniquement en regard des IgG. Curieusement, la zone d'exclusion des CD45 dépasse celle des interactions Fc $\gamma R-\lg G$, et on y détecte de l'actine, des intégrines activées et la vinculine, une protéine liant l'actine, y est aussi recrutée. Dès lors, se pose la question du mécanisme par lequel l'engagement des Fc $\gamma R$ active, à distance, les intégrines? Les auteurs démontrent que l'activation est de type inside-out, via la petite protéine $G$ Rap, qui est activée (de la forme GDP en GTP) par un facteur d'échange lui-même stimulé par l'engagement des FcyR. Les intermédiaires de cette voie Rap restent encore à identifier. Mais, comme le dit si bien Pierre Joliot «La recherche doit avant tout être un jeu et un plaisir». Ainsi, déterminer les intermédiaires de cette voie de signalisation pourrait être un axe de recherche enthousiasmant.

Les intégrines activées vont, d'une part, amplifier l'efficacité de cette étape de la phagocytose initiée par la spécificité de la reconnaissance entre le $\mathrm{F} c \gamma \mathrm{R}$ et son ligand, et, d'autre part, étendre la région d'exclusion du CD45. Elles le font en se regroupant, ce qui a quatre conséquences importantes. La première est de favoriser le regroupement (clustering) de Fc $\gamma R$ au niveau de la synapse phagocytaire; la seconde d'interagir, via leur partie extracellulaire, avec la paroi du pathogène, afin de faciliter les interactions Fc $\gamma R / \lg$; la troisième de contribuer au recrutement intracellulaire de la taline et de la vinculine, molécules servant d'intermédiaires entre la partie intracellulaire des intégrines et le réseau de filaments d'actine. Elles vont aussi favoriser la polymérisation de l'actine en activant le complexe Arp2/3; enfin, l'activation des intégrines et leur association au réseau du cytosquelette permettent l'accumulation et le maintien des molécules CD45 en dehors de la synapse phagocytaire. La taille de la zone de déplétion dépend de la distance entre deux éléments opsonisés (spots d'lgG dans le système expérimental utilisé). Quand ces éléments sont distants de $6 \mu \mathrm{m}$, une zone d'exclusion se forme pour chaque spot d'IgG, alors que si les éléments sont distants de $2 \mu \mathrm{m}$ ou moins, les zones d'exclusion fusionnent pour n'en former qu'une, plus large.

Ces découvertes faites par Spencer $A$. Freeman et al. [2] permettent d'établir la séquence d'évènements conduisant à exclure CD45 par restriction 
stérique, puis, via l'activation des Src kinases, à coordonner la réponse phagocytaire. Ces chercheurs montrent pour la première fois la mise en place d'une barrière de diffusion au sein de la synapse phagocytaire pour exclure CD45 et permettre l'initiation de la phagocytose. Mais cette barrière de diffusion progressive est-elle stricte où laisse-t-elle passer certaines molécules autres que CD45 ? II faudra continuer de chercher pour le savoir.
L'ensemble de ces résultats exposent une coordination fine de la mise en place de la phagocytose ce qui représente des avancées majeures dans la compréhension des mécanismes d'initiation de celle-ci. $\diamond$

The diffusional barrier, a new window for exclusion

\section{LIENS D'INTÉRÊT}

Les auteurs déclarent n'avoir aucun lien d'intérêt concernant les données publiées dans cet article.

\section{RÉFÉRENCES}

1. Flannagan RS, Jaumouillé V, Grinstein S. The cell biology of phagocytosis. Annu Rev Pathol 2012 ; 7 : 61-98.

2. Freeman $S A$, Das R, Grinstein $S$, et al. Integrins form an expanding diffusional barrier that coordinates phagocytosis. Cell 2016; $164: 128-40$.

3. Goodridge HS, Reyes CN, Weiss A, et al. Activation of the innate immune receptor Dectin-1 upon formation of a phagocytic synapse. Nature $2011 ; 472$ : 471-5.

4. Trimble WS., Grinstein S. Barriers to the free diffusion of proteins and lipids in the plasma membrane. J Cell Biol $2015 ; 208$ : 259-71.

5. Ewers H, Tada T, Choquet D, et al. A septin-dependent diffusion barrier at dendritic spine necks. PLoS One 2014 ; 9 : el113916.

\section{NOUVELLE}

\section{Réponse cellulaire à l'adénovirus 5}

\section{Une réponse aux dommages à l'ADN revisitée}

Baptiste Nguyen ${ }^{1}$, Georges Farkouh ${ }^{1}$, Séverine Raquin ${ }^{1}$, Karim Benihoud ${ }^{2}$

Le rôle antiviral du complexe MRN

La réplication, l'héritabilité et le maintien fidèle de I'ADN génomique sont indispensables à la vie. Les mécanismes de réparation de I'ADN, qui sont présents de la levure à l'homme, permettent de protéger le génome, notamment en réagissant aux cassures double brin (CDB) de I'ADN, qui peuvent mener à une instabilité génomique et à la tumorigenèse. Les cassures double brin sont détectées par le complexe MRN composé des protéines MREll, RAD50 et NBS1 (Figure IA). Ce complexe induit la formation de foyers de réponses aux dommages à I'ADN (RDA), en recrutant et activant la kinase ataxia telangiectasia mutated (ATM). Le signal est

Cette Nouvelle fait partie d'une série de 15 Nouvelles rédigées par les étudiants du Master «Biologie Santé » de l'université Paris-Saclay qui paraîtront dans les numéros $6-7,8-9$ et 10 (2016) de médecine/sciences. ensuite transmis via la phosphorylation des histones H2AX ( $\gamma \mathrm{H} 2 \mathrm{AX}$ ) par ATM, et aboutit au recrutement de la protéine d'échafaudage MDCl (mediator of DNA-damage checkpoint 1). Celleci recrute alors différentes protéines, dont MRN et ATM, propageant et amplifiant les marques $\gamma \mathrm{H} 2 \mathrm{AX}$ sur plusieurs mégabases du génome [1]. La fin du processus de réponse aux dommages à I'ADN se caractérise par une phosphorylation globale de substrats (dans tout le noyau). Celle-ci est assurée par les kinases effectrices CHKl (checkpoint kinase 1) et CHK2, qui phosphorylent p53 et induisent l'arrêt du cycle cellulaire via l'activation de la transcription de p2l, un inhibiteur des kinases cycline-dépendantes (CDK). Le processus peut aussi se conclure par la réparation de I'ADN, la sénescence ou l'apoptose.
${ }^{1}$ Ml Biologie Santé, Université Paris-Saclay, 91405 Orsay, France ;

${ }^{2}$ Vectorologie et thérapeutiques anticancéreuses, UMR 8203 CNRS, Université Paris-Sud, Institut Gustave Roussy, Université ParisSaclay, 94805 Villejuif, France. baptiste.nguyen@u-psud.fr georges.farkouh@u-psud.fr severine.raquin@u-psud.fr. karim.benihoud@gustaveroussy.fr

Les virus constituent une menace pour la viabilité cellulaire, mais également pour l'intégrité du génome cellulaire. Parmi ces virus, l'adénovirus possède un génome linéaire de $36 \mathrm{~kb}$ qui est délivré dans le noyau de la cellule hôte pour permettre sa réplication. Les extrémités de ce génome ressemblent à des cassures double brin, elles devraient donc être des cibles détectées par les complexes MRN. Cependant, en 2002, Stracker et al. remarquent que deux protéines précoces (ainsi appelées parce que traduites à partir de transcrits produits très rapidement après l'infection) de l'adénovirus 5 ( $A d 5), \varepsilon 1 B-55 K$ et $\varepsilon 4-0 R F 3$, inactivent les complexes MRN. Ainsi, la liaison de ElB-55k à MREll (avec l'aide d'une autre protéine adénovirale, ع4-0RF6) permet la dégradation de celle-ci par le protéasome, tandis que la protéine $\varepsilon 4-0 R F 3$ modifie la localisation 